\title{
ON MEASURES WITH SEPARABLE ORBIT
}

K. W. TAM

Let $G$ be a locally compact Hausdorff group. Let $C_{0}(G)$ denote the set of all complex-valued continuous functions on $G$ such that for each $\epsilon>0$, there exists a compact subset $K$ of $G$ such that $|f(x)|<\epsilon$ for all $x \notin K$. Let $C_{00}(G)$ be the subset consisting of functions in $C_{0}(G)$ with compact support. Suppose $V(G)$ be the set of all real or complex regular Borel measures on $G$ which are finite on compact sets. The subset $M(G)$ which have finite total variation is the dual of $C_{0}(G)$. For $\mu \in V(G)$ and $s \in G$ let $\mu_{s}$ be the measure in $V(G)$ defined by $\mu_{s}(E)=\mu\left(E s^{-1}\right)$ for each Borel set $E$. A measure $\mu$ is said to have separable orbit if there is a countable subset $C \subseteq G$ with the property that $s \in G$ and $\epsilon>0$ there exists a $c \in C$ such that $\left|\mu_{s}-\mu_{c}\right|(G)$ $=\left\|\mu_{s}-\mu_{c}\right\|<\epsilon$.

In Theorem 2 of $[2$, p. 570$]$, it was proved that a measure with separable orbit is absolutely continuous if $G$ is second countable. We shall give a proof of this result without the second countability of $G$. When $G$ is not $\sigma$-compact, $\mu \in V(G)$ has separable orbit if and only if $G$ contains an open-closed subgroup $H$ whose index is at most countable and $\mu$ on each coset of $H$ is some multiple of the Haar measure on that set.

We begin with the following lemma which has been proved in $[2$, p. 570] under the condition that $G$ is second countable. We give a different argument without this restriction.

Lemma. For every $\mu$ in $V(G)$ which has separable orbit, $\lim _{s \rightarrow t}\left\|\mu_{s}-\mu_{t}\right\|$ $=0$. Thus if $\mu$ is in $M(G)$, the function $s \rightarrow \mu_{s}$ is continuous.

Proof. For each integer $m>0$,

$$
G=\bigcup_{c \in C}\left\{x \in G:\left\|\mu_{x}-\mu_{c}\right\| \leqq 1 / m\right\} .
$$

Let $x_{\alpha}$ be in $S_{c}=\left\{x:\left\|\mu_{x}-\mu_{c}\right\| \leqq 1 / m\right\}$ and converge to $x$. Since the function $f\left(x_{\alpha} s\right)$ converges to $f(s)$ uniformly with every $f$ in $C_{00}(G)$,

$$
\lim \int f(s) d \mu_{x_{\alpha}}(s)=\lim \int f\left(s x_{\alpha}\right) d \mu(s)=\int f(s) d \mu_{x}(s) .
$$

Thus $\left|\int f(s) d\left(\mu_{x}-\mu_{c}\right)\right|=\left|\lim \int f(s) d\left(\mu_{x_{\alpha}}-\mu_{c}\right)\right| \leqq \lim \sup \left\|\mu_{x_{\alpha}}-\mu_{c}\right\|\|f\|_{\infty}$ $\leqq\|f\|_{\infty} / m$ for all $f \in C_{00}(G)$. So $\left\|\mu_{x}-\mu_{c}\right\| \leqq 1 / m$, and $S_{c}$ is closed. As

Received by the editors January 29, 1969 and, in revised form March 31, 1969. 
$G$ is locally compact Hausdorff, it is of second category and there is a $c_{m}$ so that $S_{m}=\left\{x:\left\|\mu_{x}-\mu_{c_{m}}\right\| \leqq 1 / m\right\}$ has interior. Then there exists a neighborhood $U_{m}$ of $e$, the identity of $G$, such that $x_{m} U_{m} \subseteq S_{m}$. Now for each $s \in U_{m}$, we have

$$
\left\|\mu_{s}-\mu\right\|=\left\|\mu_{s x_{m}}-\mu_{x_{m}}\right\| \leqq\left\|\mu_{s x_{m}}-\mu_{c_{m}}\right\|+\left\|\mu_{c_{m}}-\mu_{x_{m}}\right\| \leqq 2 / m .
$$

Thus $\lim _{s \rightarrow e}\left\|\mu_{s}-\mu\right\|=0$.

THEOREM 1. Every measure $\mu$ in $V(G)$ which has a separable orbit is absolutely continuous.

Proof. For every compact set $K$ in $G$ we have

$$
\left|\mu_{s}-\mu_{t}\right|(K) \leqq \| \mu_{s}-\mu_{t}|| .
$$

Hence $\mu_{t}(K)$ is continuous as a function of $t$ and $\mu$ is absolutely continuous [3, p. 230].

If $G$ is not $\sigma$-compact, then it has an open-closed subgroup $K$ such that $G / K$ is uncountable [1, p. 34]. Every $\mu \in M(G)$ is supported on a countable union of cosets of $K$. So the orbit of $\mu$ cannot be separable unless $\mu=0$. We wish to thank the referee for the following:

Theorem 2. Suppose $G$ is not $\sigma$-compact. Then $\mu \in V(G)$ has separable orbit if and only if there is an open-closed subgroup $H$ of $G$ whose index in $G$ is at most countable, and such that the restriction of $\mu$ to each coset of $H$ is a multiple of the Haar measure on that set. In particular, if $\mu$ has separable orbit, then its orbit is at most countable.

Proof. Let $H$ be the set $\left\{s \in G:\left\|\mu_{s}-\mu\right\|<\infty\right\}$. For any $s, t \in H$, $\left\|\mu_{s t^{-1}}-\mu\right\| \leqq\left\|\mu_{s}-\mu_{t}\right\| \leqq\left\|\mu_{s}-\mu\right\|+\left\|\mu_{t}-\mu\right\|<\infty$. Hence $H$ is a subgroup. By the Lemma, $H$ is open and thus closed. Since the orbit of $\mu$ is separable, $H$ has at most countable index in $G$. Moreover, $H$ is not $\sigma$-compact. For all $t \in H, \mu_{t}-\mu \in M(G)$ has a separable orbit. So $\mu_{t}-\mu=0$ and $\mu$ is invariant under translation by elements in $H$. Thus $\mu$ on coset of $H$ must be a multiple of Haar measure on that set. The converse is clear.

THEOREM 3. If $G$ is $\sigma$-compact, and $\mu \in V(G)$ is such that $\lim _{s \rightarrow e}\left\|\mu_{s}-\mu\right\|=0$, then $\mu$ has separable orbit. In particular, $\mu \in M(G)$ is absolutely continuous if and only if it has separable orbit.

Proof. Again let $H$ be the set $\left\{s \in G:\left\|\mu_{s}-\mu\right\|<\infty\right\}$. As in the proof of Theorem 2, $H$ is an open-closed subgroup. Since $G$ is $\sigma$-compact, it has the Lindelöf property. The index of $H$ in $G$ is at most countable, Now the map $s \rightarrow \mu_{s}-\mu$ on $H$ is continous in the total variation norm. So the set $\left\{\mu_{s}-\mu: s \in H\right\}$ is $\sigma$-compact and thus separable. It follows 
that the orbit of $\mu$ under translation by elements of $H$ is separable. Since the index of $H$ in $G$ is at most countable, the orbit of $\mu$ is separable.

\section{BIBLIOGRAPHY}

1. E. Hewitt and K. Ross, Abstract harmonic analysis, Springer, Berlin, 1963.

2. R. Larsen, Measures with separable orbit, Proc. Amer. Math. Soc. 19 (1968), 569-572.

3. W. Rudin, Measures algebras on abelian groups, Bull. Amer. Math. Soc. 65 (1959), 227-247.

Portland State University 